\title{
Naive Bayes applied impacts harmonic analysis in industrial electrical systems
}

\author{
Waterloo Ferreira da Silva ${ }^{1}$, Rildo de Mendonça Nogueira ${ }^{1}$, Anderson Castro ${ }^{1}$, Ádamo L. Santana ${ }^{2}$, Maria Emília de Lima \\ Tostes $^{2}$ \\ ${ }^{1}$ Programa de Pós Graduação em Engenharia Elétrica - PPGEE, Universidade Federal do Pará - UFPA. - Belém-PA, Brasil. \\ (waterloof@gmail.com, rildonogueira@hotmail.com, anderson.c@samsung.com) \\ ${ }^{2}$ Instituto de Tecnologia - ITEC, Universidade Federal do Pará -UFPA, Belém-PA,Brasil (tostes@ufpa.br,adamo@ufpa.br)
}

\begin{abstract}
The research of this paper was supported by a technique that made use of a data-mining tool and probability theory to group the data and find the relationship in each set. The Naive Bayes technique sort the data by representing them with directed acyclic graphs in which node represent random variables and the arcs represent the direct probabilistic dependencies between each other. The sorting data method is very important when it is necessary to identify the influence, occurrence and importance of a data among a set of data (database). This data mining technique applied at this paper was used to determine the impact of the harmonic distortion of the current due the nonlinear loads present during the production process of a manufacturing facility located at Manaus industrial park. The process of data collecting was conducted by a measurement campaign during a week where the voltage and current of each production process and the sub process that compose the main like the temperature control process were collected and stored for more detailed analysis. The parameters used to analyze the energy quality were based on the module 8 from the Energy Distribution Proceedings (PRODIST), which rules the energy quality at the distribution network. The results of this paper can be used to conduct the countermeasures necessary to fix the current harmonic distortion influence at the voltage and consequently the active power consume reduction of the overall company.
\end{abstract}

Keywords: Naïve Bayes, Harmonics, Data Mining, Power Systems.

\section{Naive Bayes aplicados en el análisis de impactos armónicos en los sistemas eléctricos industriales}

\section{RESUMEN}

La investigación que se presenta en el trabajo se apoya en técnicas estadísticas como la prospección de datos y la teoría de las probabilidades lo que permite agrupar datos y encontrar una relación en cada uno de los grupos. La técnica de Naive Bayes agrupa los datos en grafos acíclicos en el cual los nodos representan variables aleatorias y los arcos representan dependencias probabilísticas directas entre cada una. El método de agrupación es muy importante cuando es necesario identificar la influencia, presencia e importancia de los datos en un conjunto de datos (base de datos). La técnica de prospección de datos aplicada en el presente trabajo permite determinar el impacto de la distorsión armónica de corriente debido a la presencia de cargas no lineales presentes en el proceso producción de una instalación manufacturera localizada en el parque industrial de Manaos. El proceso de recolección de datos se realizó durante una semana de medición continua del voltaje y la corriente de cada proceso y subprocesos de producción que la componen, así como el control de la temperatura, dichos datos fueron almacenados para su posterior análisis. Los parámetros utilizados para analizar la calidad de la energía se basan en el módulo 8 de los procedimientos de energía "PRODIST" que regula la calidad de la energía en las redes de distribución. Los resultados de este trabajo se pueden utilizar para tomar las medidas necesarias para corregir la influencia de la distorsión de la corriente de armónicos de la tensión y por lo tanto la reducción del consumo de energía activa de toda la empresa.

Palabras clave: Naive Bayes, armónicos, prospección de datos, sistemas de potencia.

\section{INTRODUCCIÓN}

Las formas existentes de la energía representan actualmente un incentivo para los países en desarrollo al impactar directamente en la sociedad en todos sus aspectos. Una de esas formas es la energía eléctrica que mueve muchos segmentos, ya sea para consumo o para la producción [1].
La disponibilidad de electricidad incide en la calidad de vida de las poblaciones. En el primer momento se despliega un sistema de distribución de energía eléctrica, la población local de inmediato comienza a aparecer con numerosos beneficios, tanto en el confort, la creación de nuevos puestos de trabajo y por lo tanto en el proceso general de desarrollo.

ITEGAM - JETIA Vol. 01, Nº. 02. Junho de 2015. Manaus - Amazonas, Brasil. ISSN 2447-0228 (ONLINE). 
Waterloo Ferreira da Silva et al. / ITEGAM-JETIA Vol. 01, N ${ }^{\mathrm{o}}$ 02, pp.45-54. Junho, 2015.

Esta necesidad llevó al desarrollo de la electrónica de potencia, las nuevas tecnologías que involucran materiales semiconductores de características no lineales comenzaron a ser usados para lograr las soluciones de las industrias. Así, los problemas con los armónicos llegaron a ser pronunciados [2].

Los armónicos del sistema de potencia se definen como tensión y corriente sinusoidal con frecuencias que son múltiplos enteros de la frecuencia principal (o primario) generada [3].

Tenga en cuenta que el estricto cumplimiento de los límites recomendados por las normas no elimina necesariamente los problemas, sobre todo cuando los sistemas están operando cerca de los límites.

En general, este comportamiento no lineal puede ser observado por la no conformidad de la forma de la onda de la corriente con relación a la forma de la onda de la carga de alimentación [4] [5].

Hay varios estándares y recomendaciones de diversos organismos internacionales sobre los límites de armónicos en sistemas eléctricos. Todas estas recomendaciones se desarrollan con la ayuda de numerosos fabricantes de equipos, empresas de servicios energéticos y las instituciones de investigación. La. IEEE Std 519-1992 como prácticas y requisitos para el control de armónicos en sistemas eléctricos de potencia recomendados, propone un reparto de responsabilidades para el mantenimiento de los armónicos en el PAC (Punto de acoplamiento común), donde los consumidores deben asegurarse de que las corrientes armónicas sean limitadas y los distribuidores, a su vez, deben garantizar los límites de distorsión armónica de la tensión [6].

La IEC 61000-2-2 limita la distorsión armónica de tensión para los consumidores con tensión de alimentación entre $240 \mathrm{~V}$ y $450 \mathrm{~V}$ para sistemas trifásicos o sistema monofásicos que están operando con una frecuencia nominal de $50 \mathrm{~Hz}$ o $60 \mathrm{~Hz}$ [7].

La norma IEC 61000-4-7 especifica las directrices necesarias para el proyecto y construcción de medidores de distorsión armónica. De acuerdo con esta norma, THD los metros están compuestos básicamente de: circuito de entrada con filtros anti-aliasing, convertidores A / D, unidad de sincronización y un procesador de transformada de Fourier discreta responsable de proporcionar los coeficientes de Fourier. En los aspectos de metrología, la IEC 61000-4-7 define dos clases de precisión para la medición de THD. La clase I se utiliza cuando se necesitan mediciones de alta precisión de exactitud. Se recomiendan los instrumentos de clase II para la mayoría de aplicaciones generales [8].

La norma IEC 61000-4-30, publicada en 2003, que tiene como objetivo poner fin a las incertidumbres con respecto a la forma de medir los parámetros de calidad, también conocido como protocolos de medición [9]. En Brasil, la Agencia Nacional de Energía Eléctrica (ANEEL) desarrolló los procedimientos de distribución de energía (PRODIST) [10]. La revisión actual de PRODIST entró en vigor el 1 de enero 2010 Entre los principales

objetivos de PRODIST esta asegurarse de que los sistemas de distribución operen con seguridad, la eficiencia, la calidad y la fiabilidad requeridos. El Módulo 8 PRODIST regula la calidad de la energía en las redes de distribución.

Dentro de la inyección de armónicos en la red eléctrica, PRODIST define los límites en las tablas I y II.

TABLA I - valores globales de las distorsiones armónicas totales

\begin{tabular}{c|c}
\hline $\begin{array}{c}\text { Tensión nominal en las } \\
\text { barras }\end{array}$ & $\begin{array}{c}\text { Distorsión armónica total } \\
\text { de la tensión }(\%)\end{array}$ \\
\hline $\mathrm{Vn} \leq 1 \mathrm{kV}$ & 10 \\
\hline $1 \mathrm{kV}<\mathrm{Vn} \leq 13,8 \mathrm{kV}$ & 8 \\
\hline $13,8 \mathrm{kV}<\mathrm{Vn} \leq 69 \mathrm{kV}$ & 6 \\
\hline $69 \mathrm{kV}<\mathrm{Vn} \leq 230 \mathrm{kV}$ & 3 \\
\hline
\end{tabular}

TABLA II - niveles de referencia para la distorsión armónica individual de la tensión

\begin{tabular}{|c|c|c|c|c|c|}
\hline \multirow{2}{*}{\multicolumn{2}{|c|}{ Orden armónico }} & \multicolumn{4}{|c|}{$\begin{array}{c}\text { Distorsión armónica individual } \\
\text { de la tensión [\%] }\end{array}$} \\
\hline & & \multirow{2}{*}{$\begin{array}{l}\mathrm{Vn} \leq \\
1 \mathbf{k V} \\
7,5\end{array}$} & \multirow{2}{*}{$\begin{array}{c}1 \mathrm{kV} \\
<\mathrm{Vn} \leq \\
13,8 \mathrm{kV}\end{array}$} & \multirow{2}{*}{$\begin{array}{c}13,8 \\
\mathbf{k V} \\
<\mathbf{V n} \leq \\
\mathbf{6 9} \mathbf{k V} \\
4,5\end{array}$} & \multirow{2}{*}{$\begin{array}{l}<9 \mathbf{k V} \\
<\mathbf{V n} \leq \\
230 \\
\mathbf{K v} \\
2,5\end{array}$} \\
\hline \multirow{9}{*}{$\begin{array}{l}\text { Impares } \\
\text { no } \\
\text { múltiplos } \\
\text { de } 3\end{array}$} & 5 & & & & \\
\hline & 7 & 6,5 & 5 & 4 & 2 \\
\hline & 11 & 4,5 & 3,5 & 3 & 1,5 \\
\hline & 13 & 4 & 3 & 2,5 & 1,5 \\
\hline & 17 & 2,5 & 2 & 1,5 & 1 \\
\hline & 19 & 2 & 1,5 & 1,5 & 1 \\
\hline & 23 & 2 & 1,5 & 1,5 & 1 \\
\hline & 25 & 2 & 1,5 & 1,5 & 1 \\
\hline & $>25$ & 1,5 & 1 & 1 & 0,5 \\
\hline \multirow{5}{*}{$\begin{array}{c}\text { Impares } \\
\text { múltiplos } \\
\text { de } 3\end{array}$} & 3 & 6,5 & 5 & 4 & 2 \\
\hline & 9 & 2 & 1,5 & 1,5 & 1 \\
\hline & 15 & 1 & 0,5 & 0,5 & 0,5 \\
\hline & 21 & 1 & 0,5 & 0,5 & 0,5 \\
\hline & $>21$ & 1 & 0,5 & 0,5 & 0,5 \\
\hline \multirow{7}{*}{ Pares } & 2 & 2,5 & 2 & 1,5 & 1 \\
\hline & 4 & 1,5 & 1 & 1 & 0,5 \\
\hline & 6 & 1 & 0,5 & 0,5 & 0,5 \\
\hline & 8 & 1 & 0,5 & 0,5 & 0,5 \\
\hline & 10 & 1 & 0,5 & 0,5 & 0,5 \\
\hline & 12 & 1 & 0,5 & 0,5 & 0,5 \\
\hline & $>12$ & 1 & 0,5 & 0,5 & 0,5 \\
\hline
\end{tabular}

A pesar de la legislación vigente no penaliza a la industria par la generación de corrientes armónicas en el sistema eléctrico, ya hay señales de que la ANEEL pronto anunciará una nueva legislación para evaluar los impactos de las industrias, como se cita en la nota técnica 0083/2012-SRD / ANEEL [11] donde se refiere a la norma de Australiana que impone valores de referencia para la distorsión armónica de la de tensión (que deben observarse por el distribuidor) y las corrientes armónica de los dispositivos conectados a la red (que deben observarse por el consumidor). 
Waterloo Ferreira da Silva et al. / ITEGAM-JETIA Vol. 01, N ${ }^{\mathrm{o}}$ 02, pp.45-54. Junho, 2015.

pico de tensión (puede conducir a la avería del aislamiento) aumentando; aumentando los valores eficaces (rms) (conduce a una mayor pérdida de potencia) y el aumento en el espectro de frecuencia de corriente y tensión (vibración adicional y desgaste mecánico) [12] [13]. Debido al flujo de corrientes armónicas se produce el calentamiento adicional en las líneas de alimentación, alternadores y motores, transformadores, por lo tanto, causan daños en el sistema de corrección del factor de potencia. Además, los problemas inesperados en los dispositivos de protección, manejo incorrecto en equipos sensibles electrónicos (computadoras, mando y control, etc) y los errores en los valores de los equipos de medición (por ejemplo, balanzas industriales, equipos de dosímetros electrónicos, etc) [13].

Teniendo como objetivo evaluar el impacto de los armónicos en una fábrica de electrónicos en el PIM y anticiparse a la próxima legislación que entrara en vigor, fue definido junto con la industria y los investigadores la metodología a aplicar en el presente trabajo: Se instalaron 5 medidores de la calidad de la energía durante siete días en diferentes circuitos de la industria, recolectar datos, conversión de datos, aplicar técnicas estadísticas de prospección de datos y evaluar los resultados.

Por lo tanto, este artículo presenta una técnica de análisis de datos utilizando redes bayesianas más específicamente con Naive Bayes para identificar el impacto de los componentes de corrientes armónicas individuales de cargas no lineales de los equipos electrónicos de THDv de la tensión a la entrada de una industria del Polo Industrial de manaos.

\section{MATERIALES Y MÉTODOS}

La prospección de datos es una parte integral del "descubrimiento de conocimientos en bases de datos" (kdd knowledgedicovery in database), que es el proceso general de liberación de datos globales en información útil, como se muestra en la figura 1. este proceso consiste en una serie de etapas de transformación, desde el pre-procesamiento de los datos hasta el post-procesamiento de los resultados de la prospección de datos [17]. investigación.

Esta fue la metodología elegida para llevar a cabo la

Los datos de entrada pueden ser almacenados en una variedad de formatos (archivos planos, hojas de cálculo o tablas relacionas) y pueden permanecer en un repositorio de datos central o ser distribuido en múltiple locales [17].

Fue elegido para almacenar los datos en tablas relacionadas de una base de datos.

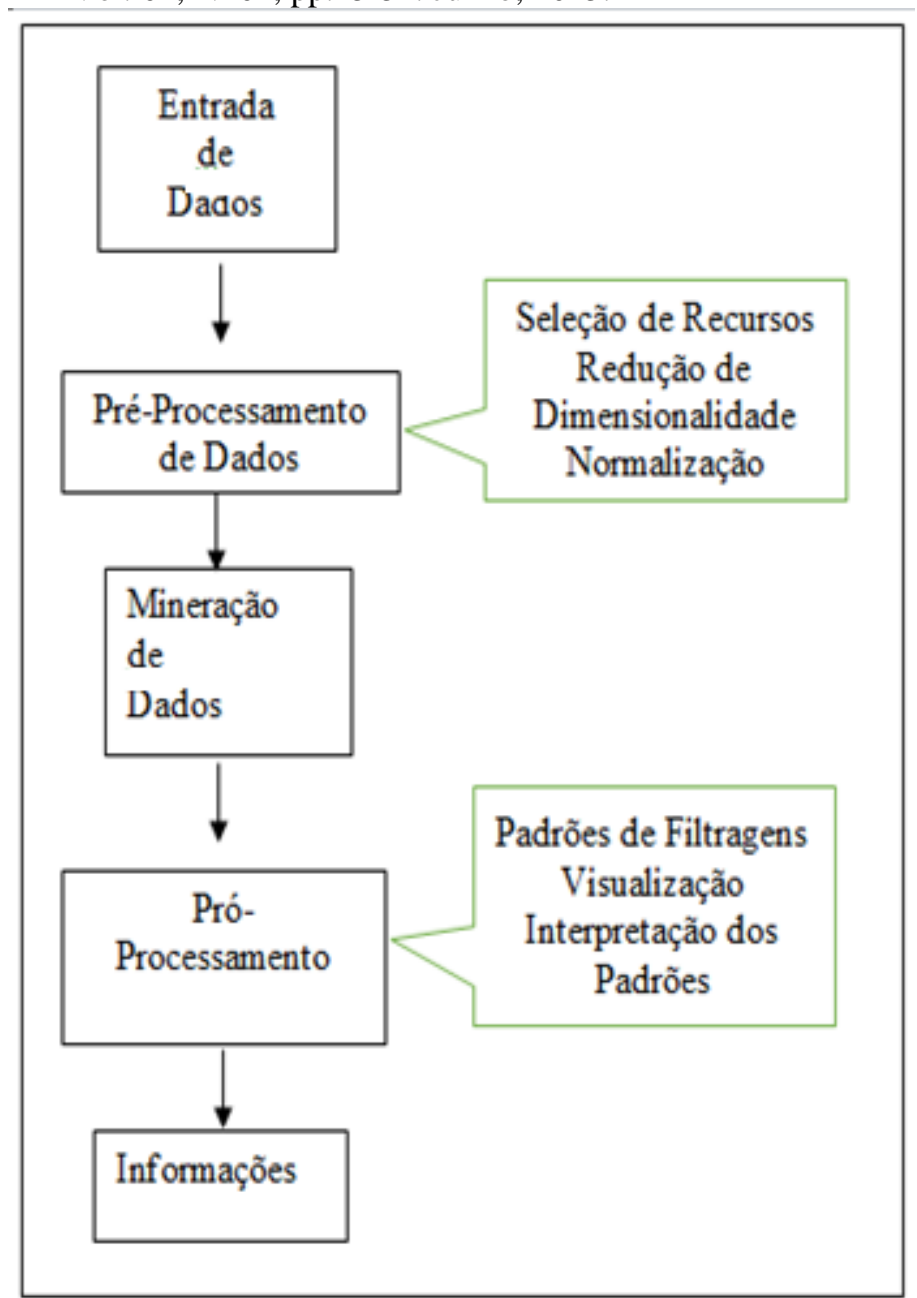

Figura. 1 Proceso de descubrimiento del conocimiento en bases de datos (KDD).

El propósito de pre-procesamiento es transformar la entrada de datos en bruto en un formato apropiado para su posterior análisis [17]. En esta etapa se usan herramienta Weka (Waikato Environment for Knowledge Analysis) [15], que tiene una colección de algoritmos de aprendizaje automático para la solución de problemas de la prospección de datos, se realizó discretización sin supervisión de los datos de frecuencia.

Las tareas de prospección de datos se dividen generalmente en dos categorías principales:

Predicción de tareas - El objetivo de estas tareas es predecir el valor de un atributo específico basado en los valores de otros atributos. El atributo que se predijo que se conoce comúnmente como la variable dependiente, mientras que los atributos utilizados para hacer la predicción son conocidos como variables independientes o explicativas [17].

La técnica elegida para el análisis fue el clasificador de Bayes simple o Naive Bayes. El Teorema de Bayes: Para llegar al teorema de Bayes, partimos del principio básico. Por lo tanto, la 
Waterloo Ferreira da Silva et al. / ITEGAM-JETIA Vol. 01, No 02, pp.45-54. Junho, 2015.

probabilidad de observar simultáneamente un evento A en un evento B viene dada por:

$$
\mathrm{P}(\mathrm{A} \cap \mathrm{B})=\mathrm{P}(\mathrm{A} / \mathrm{B}) * \mathrm{P}(\mathrm{B})
$$

Por otra parte, la probabilidad de que observe al mismo tiempo un evento A y el evento B se puede dar a través de:

$$
\mathrm{P}(\mathrm{B} \cap \mathrm{A})=\mathrm{P}(\mathrm{A} B)=\mathrm{P}(\mathrm{B} / \mathrm{A}) * \mathrm{P}(\mathrm{A})
$$

Combinando (1) y (2) tenemos:

$$
\mathrm{P}(\mathrm{A} / \mathrm{B}) * \mathrm{P}(\mathrm{B})=\mathrm{P}(\mathrm{B} / \mathrm{A}) * \mathrm{P}(\mathrm{A})
$$

Reorganizando, llegamos al teorema de Bayes,

$$
\mathrm{P}(\mathrm{A} / \mathrm{B})=\frac{\mathrm{P}(\mathrm{B} / \mathrm{A})-\mathrm{P}(\mathrm{A})}{\mathrm{P}(\mathrm{B})}
$$

Las Redes Bayesianas son herramientas poderosas para la toma y el razonamiento bajo incertidumbre. Una forma muy sencilla de las redes de Bayes son los llamados clasificadores de Naive bayesianos $[21,18,19,20]$ dedicados a la tarea de clasificación. Tales redes están compuestas por grafos acíclicos direccionados con una estructura única, tienen un nodo raíz (llamado padre), que representa el nodo de clase, y varios hijos, que corresponde a asignar nodos. Naive trabajo sobre una fuerte suposición "ingenua" de independencia entre nodos hijos en el contexto de su padre. Así, en la presencia de un conjunto de entrenamiento se debe calcular la probabilidad condicional ya que la estructura es única. Un grafo $\mathrm{G}$ es un par ordenado $\mathrm{G}=$ $(\mathrm{V}, \mathrm{A})$, donde $\mathrm{V}$ es un conjunto no vacío cuyos elementos son llamados vértices o nodos y un conjunto Ae de pares de vértices de V, cuyos elementos se denominan bordes. Por lo general V (y por tanto A) es finito, es decir, $\mathrm{V}=\{\mathrm{X} 1, \mathrm{X} 2, \ldots, \mathrm{Xn}\}$. Dos vértices conectados.

Dos vértices conectados por una arista son adyacentes. Dos aristas son adyacentes si comparten el mismo vértice. Los grafos direccionados poseen una arista del conjunto A y es representada como $(\mathrm{u}, \mathrm{v})$, donde $\mathrm{v}$ se dice que es adyacente a $\mathrm{u}, \mathrm{y}$ la arista del tipo (u, u) se denominan self-loops.

La posesión de archivo discreto en WEKA, que utiliza el software Genie 2.0 que puede ser realizado descargando http://genie.sis.pitt.edu/ y el software, Rapid Miner 5.3, que puede descargarse en http: // www.rapidminer.com, donde se aplicó la técnica de NaiveBayes de prospección de datos.

\section{INDUSTRIA ELECTRÓNICA - ESTUDIO DE CASO}

El presente estudio se desarrolló a partir del diseño del estudio de caso en una industria de la electrónica que produce una mezcla de productos manufacturados tales como televisores, computadoras de escritorio, tabletas y teléfonos, instalada en
Manaos, Polo Industrial de Manaos (PIM) con incentivos de la Zona Franca de Manaus (SUFRAMA-MFZ) por más de 30 años. Los casos piloto pueden ser realizados por varias razones no relacionadas con los criterios para la selección de los casos finales, permitiendo así que la recolección de datos para el estudio [13].

Uno de los objetivos está relacionado con la reciente aprobación de la prórroga de la Zona Franca de Manaos. Las empresas instaladas tuvieron que anticipar los incentivos permitidos para la ley de tecnologías de la información y adaptarse a la nueva legislación que ANEEL que evaluará los impactos de los armónicos de corriente de las industrias a fin de mejorar sus indicadores de calidad de la energía, permitiendo asî la recolección de datos para el estudio.

Los datos fueron recolectados en esta industria de procesos de fabricación indicador por mix de productos de alta tecnología en el que el rendimiento de estos productos genera los impactos de distorsión armónica en la red, uno de los principales problemas analizados. La duración de la recolección de datos fue discriminada por turnos de lunes a viernes en horario de la siguiente manera: el primer turno de 06:00-15:48 y el segundo de 16:00-1:30. Durante este período se estaban produciendo notebooks y tablets.

La recolección de datos fue los siguientes puntos analizados: Salida de la caja de alimentación (220/127 V) circuito que alimenta la central de aire acondicionado, circuito de la sala de compresores, circuito alimenta la prueba de notebook portátil con aproximadamente 900 notebook simultáneamente, circuito que alimenta la prueba de tabletas, con aproximadamente 140 tabletas conectadas simultáneamente. En estos puntos fueron instalados 5 metros medidores de la calidad de la energía HIOKI PW3198, como se evidencia en la Figura 2.

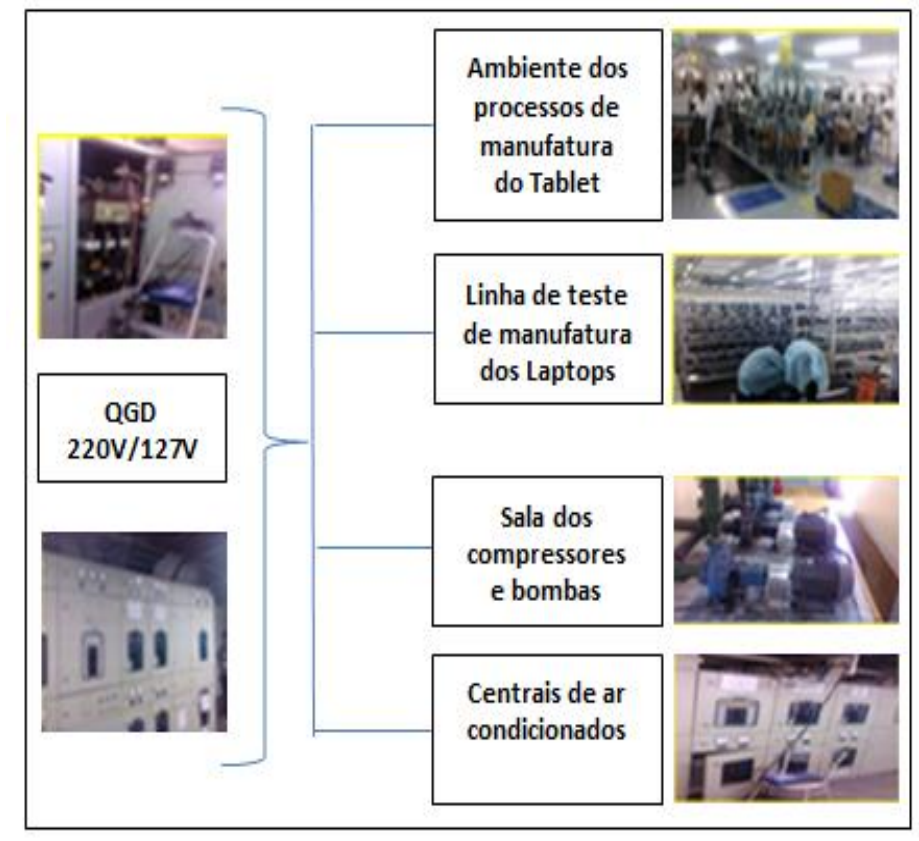

Figura 2. Puntos de instalación de Medidores de calidad de la energía. 
Las Tabla III, IV y V presentan la particularidades de las cajas de alimentación y las características técnicas y de generación de armónicos de cada circuito, tipos de cargas, así como los detalles de los períodos que se mantuvieron alimentados en la línea de burning test/tablets, tabletas, compresores y bombas / aire acondicionado central.

Tabla III. Subestación

Salida del Transformador de 1000kVA 220/127V, Alimenta disyuntor trifásico 3200A de una planta de la Industria

\begin{tabular}{|c|c|}
\hline $\begin{array}{l}\text { Cargas } \\
\text { Lineales }\end{array}$ & $\begin{array}{l}\text { Cargas de fuentes de conmutación: THDi> 93\%. } \\
\text { Armónicos: } 3 \text { (78\%), } 5 \text { (44\%), } 7 \text { (17\%) ... } 15 .\end{array}$ \\
\hline $\begin{array}{l}\text { Cargas } \\
\text { No } \\
\text { lineales }\end{array}$ & $\begin{array}{l}\text { Cargas de inversores de frecuencia: THDi> } \\
\text { 100\%. } \\
\text { Armónicos: } 5(81 \%), 7(74 \%), 11(42 \%), 13 \\
(30 \%), 17 \text { e 19, Cargas de los 5 UPSs de 80kVA } \\
\text { características de: TDHi> 30\%, Armónicos: } 5 \\
(28 \%), 7(5 \%), 11(6 \%) \text { e } 13(6 \%) \text {. } \\
\text { Cargas de lámparas fluorescente: TDHi> 50\% } \\
\text { Armónicos: 3, 5, 9 e } 11 .\end{array}$ \\
\hline
\end{tabular}

Fuente: Los autores.

Tabla IV. Compresores y bombas /aire acondicionado centralizado

01 Compresor de $75 \mathrm{CV}(55,93 \mathrm{~kW}), 01$ Bomba booster de 25 CV $(18,64 \mathrm{~kW})$ y una Bomba de Torre de $25 \mathrm{CV}(18,64 \mathrm{~kW})$

\begin{tabular}{l|l}
\hline $\begin{array}{l}\text { Circuito de Carga } \\
\text { Lineal }\end{array}$ & $\begin{array}{l}\text { con bajo impacto en la distorsión } \\
\text { armónica. }\end{array}$ \\
\hline Período & $\begin{array}{l}\text { Disponíble entre 05:30 até 01:30 de } \\
\text { lunes a viernes. No fin de semana se } \\
\text { mantiene desconectado. }\end{array}$ \\
\hline
\end{tabular}

04 Centrales de aire acondicionado, Cada Central funciona con 2 compresores de $15 \mathrm{TR}(52,75 \mathrm{~kW})$.

\begin{tabular}{ll|l}
\hline $\begin{array}{l}\text { Circuito de Carga } \\
\text { Lineal }\end{array}$ & $\begin{array}{l}\text { Bajo impacto en la distorsión } \\
\text { armónica. }\end{array}$ \\
\hline Horario & & $\begin{array}{l}\text { Disponible entre 05:30 hasta 01:30 de } \\
\text { lunes a viernes. El fin de semana esta } \\
\text { desconectado. }\end{array}$ \\
\hline
\end{tabular}

Fuente: Los autores.

\section{Tabla V. Linea burning test / tablets}

500 notebook de $65 \mathrm{~W}$ cada bifásico, fuente de conmutación de 65W, Corriente de Carga Nominal: 3,42 A, Potencia de Salida Nominal: 65W, Regulación de Salída Total: +/- 5\%, Regulación de Linea: +- 3\%, Ripple: 200mV (pico a pico). Ruído: $380 \mathrm{mV}$ (pico a pico) y THD, V $<2 \%$

\begin{tabular}{l|l}
\hline & $\begin{array}{l}\text { Cada notebook se mantiene por 1 hora y es } \\
\text { sustituido por otro. Em el período de 01:30 hasta } \\
06: 00 \text { estan conectados los notebook del día } \\
\text { anterior. Durante el fin de semana los notebooks }\end{array}$ \\
estan conectados a la red elétrica. Todos los \\
Cargas \\
Lineales \\
(UPS) de 80kVA.
\end{tabular}

10 Impresoras Laser con $57,96 \mathrm{~W}$ cada uno, 4 Balanzas Electrónicas con $15 \mathrm{~W}$ cada uno, 10 destornilladores eléctricos con motor de $40 \mathrm{~W}$ cada uno, 11 switch de red con 10,3 W cada uno, 4 Monitores de LED con $45 \mathrm{~W}$ cada uno, 01 Computador DeskTop de 352W, 1 cautín de 70W, 03 Ionizadores de Aire con 26,4 W cada uno, 8 impresora de etiqueta co $110 \mathrm{~W}$ cada una, 17 notebooks con $65 \mathrm{~W}$ cada una, 25 lámparas LED con 20W cada una, 12 dispensadores electrónicos de tornillo con $6 \mathrm{~W}$ cada uno y alrededor de 140 tablets conectados en prueba con $10 \mathrm{~W}$ cada una

\begin{tabular}{|c|c|}
\hline $\begin{array}{l}\text { Cargas } \\
\text { Lineares }\end{array}$ & 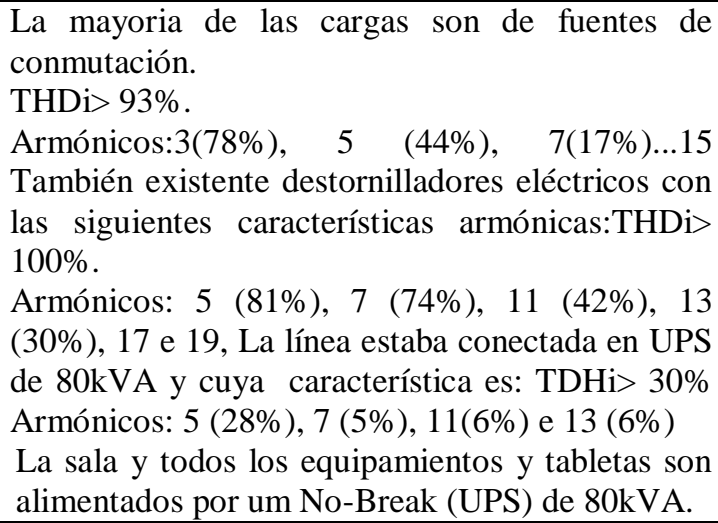 \\
\hline Perío & $\begin{array}{l}\text { 06:00 hasta 01:30 del día siguiente, de lunes a } \\
\text { viernes, la línea de equipos está apagada. En el } \\
\text { período de 01:30 a 06:00 son conectadas algunas } \\
\text { tabletas. }\end{array}$ \\
\hline
\end{tabular}

Fuente: Los autores. 


\section{4 - RESULTADOS Y DISCUSIÓN}

La Figura 3 muestra los naive bayes creados con los datos provenientes de la de medición realizada en las instalaciones de la industria, siendo registradas las siguientes magnitudes: tasa de distorsión total de armónicos del voltaje centrales.
(THDv) del secundario del transformador de entrada, corrientes armónicas de orden 3, 5, 7, 9 y 11 de los procesos burning, tablet, tablet, compresores y de aire acondicionado.

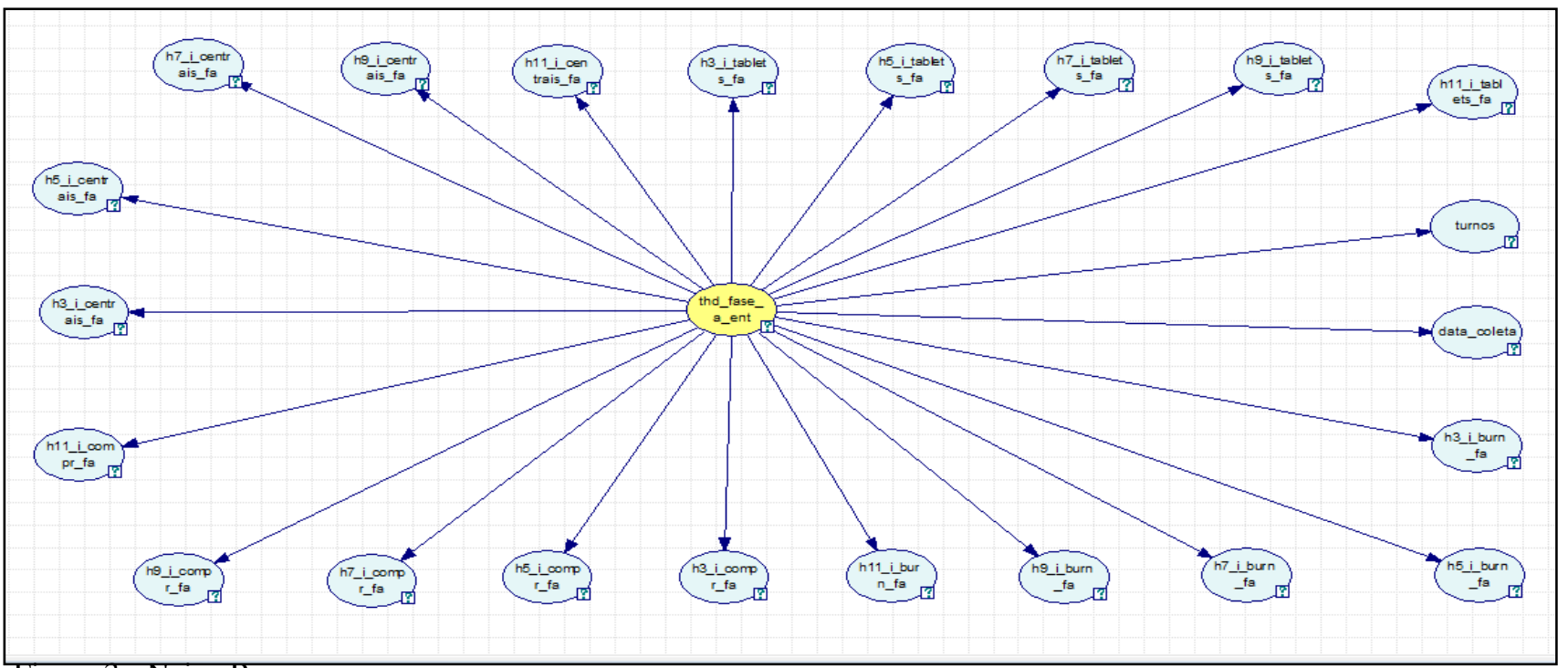

Figure 3 - Naive Bayes.

El análisis de las probabilidades obtenidas con la creación de la red mostrada en la fig. 3, se observó que las corrientes armónicas de orden 3, 5, 7 y 11 del proceso con las Tablet, la corriente armónica de orden 7 del proceso del compresor, y las corrientes armónicas de orden 5, 7 y 9 del proceso del aire centralizado tenían alta probabilidades ( $80 \%$ o mayor) asociados con valores de crecimiento de THDv de la tensión de entrada.
Analizando la Figura. 4, que muestra la densidad de probabilidad de ocurrencia de los cinco intervalos discretos de THDv en relación a la corriente del $7^{\circ}$ harmónico de la caja del compresor, se puede observar que hay una probabilidad de $88,5 \%$ de THDv teniendo un valor igual o superior a $3.085 \%$ cuando la corriente de $7^{\circ}$ armónico de la caja de compresor tiene un valor igual o mayor que $0,036 \%$.

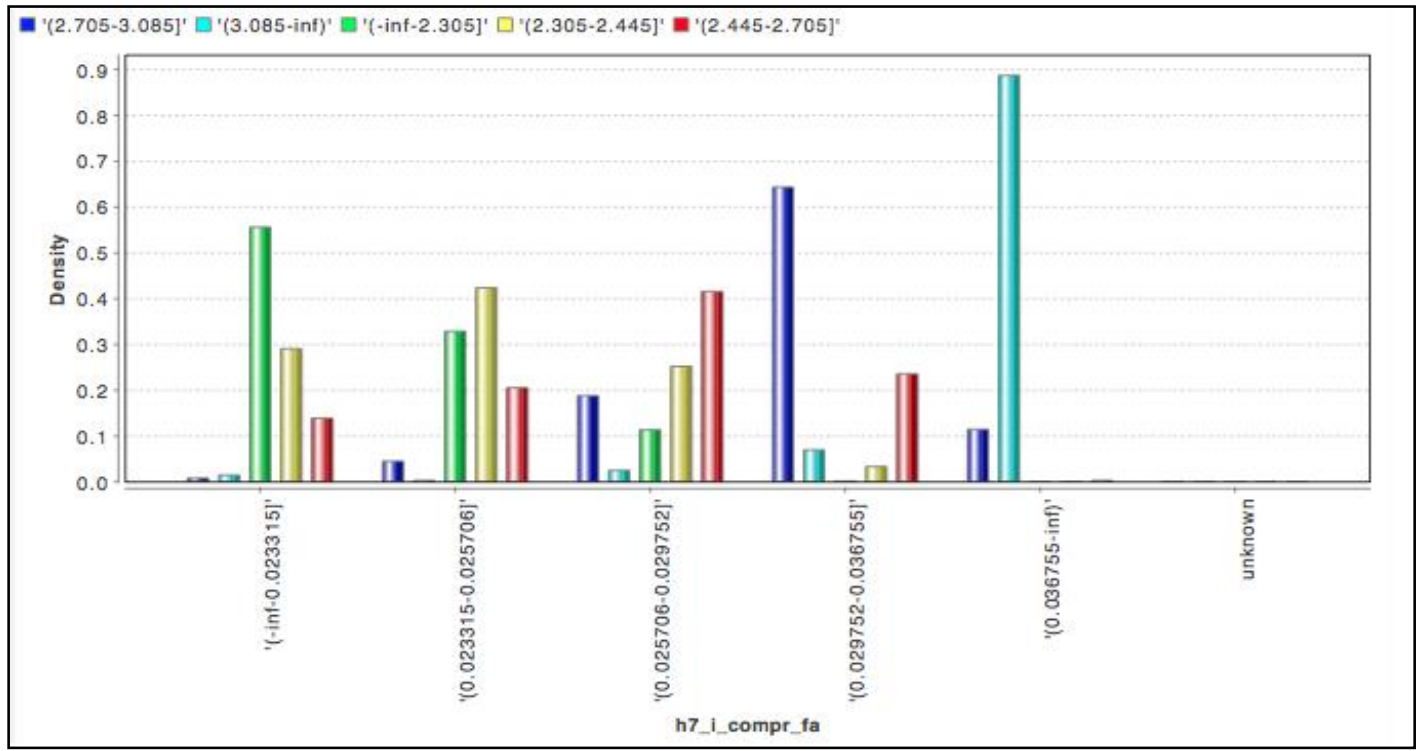

Figura 4 - Densidad de probabilidad de intervalos discretos de THDv. 
Waterloo Ferreira da Silva et al. / ITEGAM-JETIA Vol. 01, N ${ }^{\mathrm{o}}$ 02, pp.45-54. Junho, 2015.

En cuanto a la figura. La figura 5 muestra la densidad de probabilidad de ocurrencia de los cinco intervalos discretos de THDv en relación con la corriente del $5^{\circ}$ armónico de la central de aire se puede verificar que hay una probabilidad de casi el
$84,5 \%$ de THDv teniendo un valor igual o mayor que $3.085 \%$ cuando la corriente del $5^{\circ}$ armónico del proceso de la central de aire presenta un valor igual o superior a $0,008 \%$.

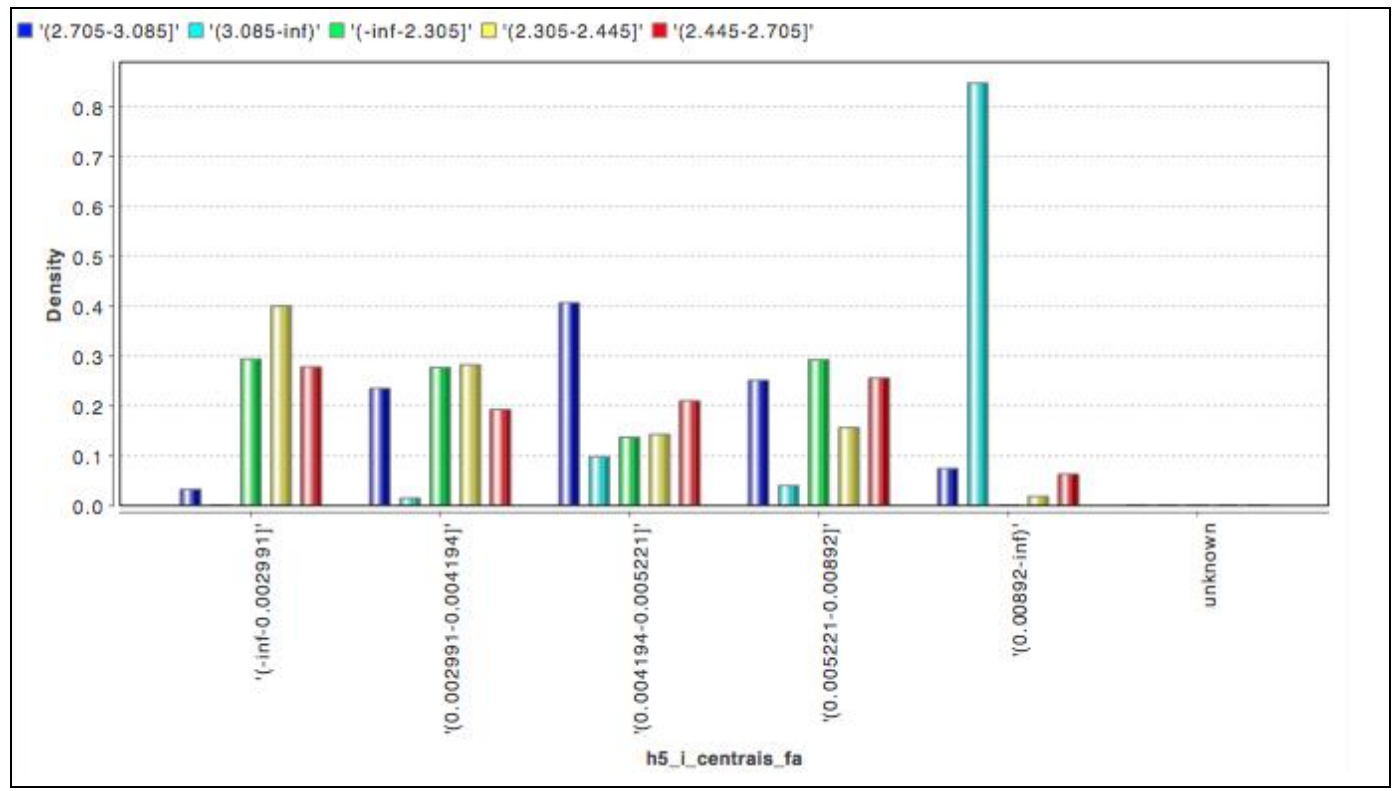

Figure 5 - Densidad de probabilidad de intervalos discretos de THDv con relación a la corriente del $5^{\circ}$ armónico.

La figura 6 muestra la densidad de probabilidad de ocurrencia de los cinco intervalos discretos de THDv respecto a la corriente del $7^{\circ}$ armónico del proceso de la central de aire y se puede ver que hay una probabilidad de casi el $87,2 \%$ de THDv teniendo un valor igual o mayor que $3.085 \%$ cuando la corriente del $7^{\circ}$ armónico del proceso de la central de aire presenta un valor igual o superior a $0,022 \%$.

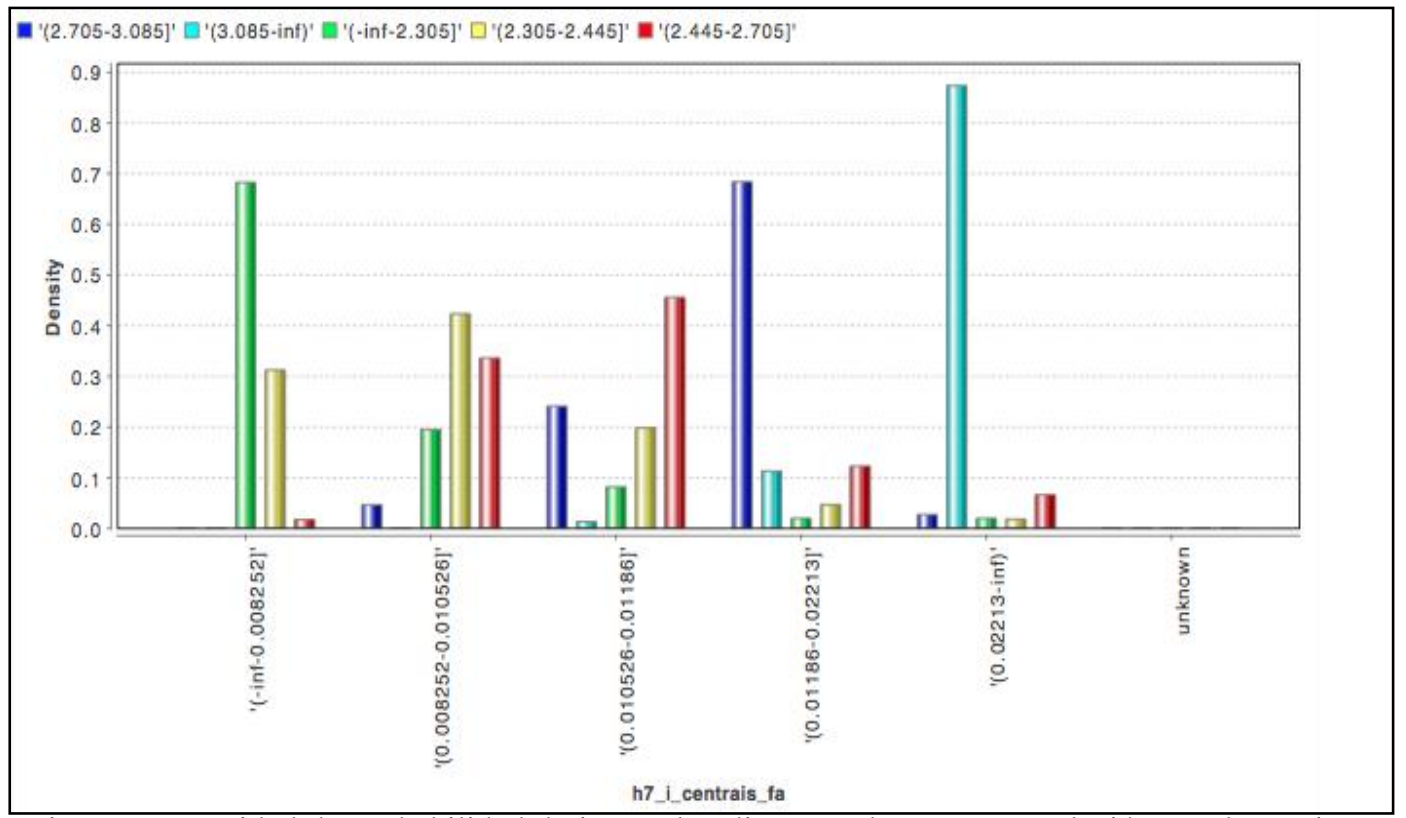

Figura 6- Densidad de probabilidad de intervalos discretos de THDv en relación con la corriente del $7^{\circ}$ armónico. 
Waterloo Ferreira da Silva et al. / ITEGAM-JETIA Vol. 01, N ${ }^{\mathrm{o}}$ 02, pp.45-54. Junho, 2015.

La figura 7 muestra la densidad de probabilidad de ocurrencia de los cinco intervalos discretos del THDv con relación a la corriente del $9^{\circ}$ armónico de la central de aire de proceso, se puede ver que hay una probabilidad de casi el $83 \%$ de THDV teniendo un valor igual o mayor que $3,085 \%$ cuando la corriente del $9^{\circ}$ armónico del proceso de la central de aire presenta un valor igual o mayor que $0,003 \%$.

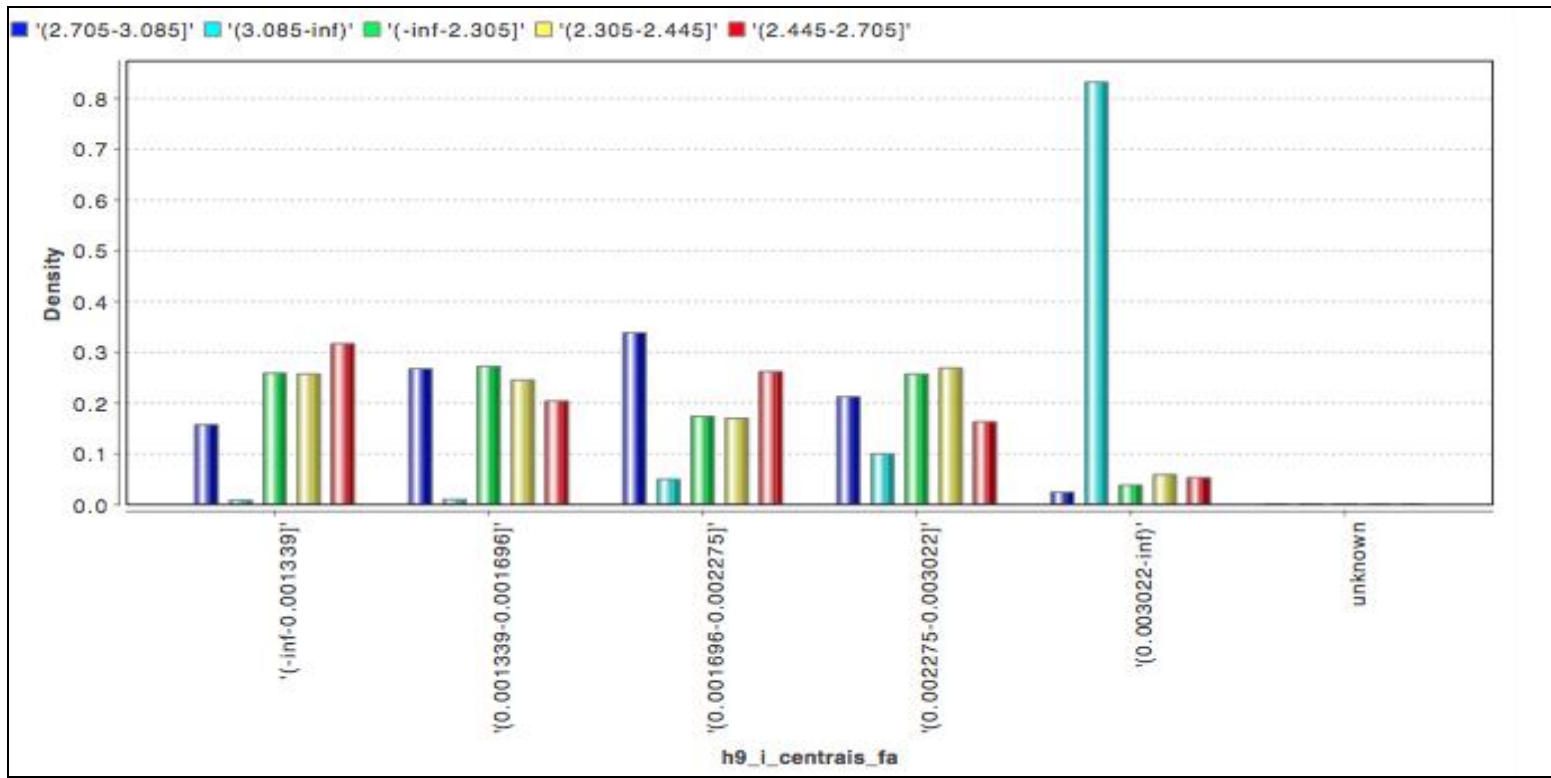

Figura 7. Densidad de probabilidad de intervalos discretos de THDv con relación a la corriente del $9^{\circ}$ armónico.

La figura 8 muestra la densidad de probabilidad de ocurrencia de cinco intervalos discretos de THDv en relación con la corriente del $3^{\circ}$ armónico del proceso de la tablet, se puede comprobar que existe una probabilidad de casi el $82,9 \%$ de
THDv teniendo un valor igual o mayor a $3.085 \%$ cuando la corriente del $3^{\circ}$ armónico del proceso de la tablet presentando un valor igual o inferior a $3,55 \%$.



Figura 8. Densidad de probabilidad de intervalos discretos de THDv en relación con la corriente del $3^{\circ}$ armónico.

La figura. 9 Muestra la densidad de probabilidad de ocurrencia de cinco intervalos discretos del THDv en relación con el $5^{\circ}$ armónico del proceso de la tablet, se puede comprobar que hay una probabilidad de casi el $82,5 \%$ del THDv teniendo un 
valor igual o mayor a $3.085 \%$ cuando la corriente del $5^{\circ}$ armónico del proceso de la Tablet presenta un valor igual o mayor a $3,05 \%$.



Figura 9. Densidad de probabilidad de intervalos discretos THDv en relación con la corriente del $5^{\circ}$ armónico.

La figura 10 muestra la densidad de probabilidad de ocurrencia de cinco intervalos discretos de THDv con relación a la corriente del $7^{\circ}$ armónico del proceso de la tablet, se puede observar que hay una probabilidad de casi el $88 \%$ de THDv teniendo un valor igual o mayor que $3,085 \%$ cuando la corriente del $7^{\circ}$ armónico del proceso de la Tablet presenta un valor igual o mayor que $4,85 \%$.

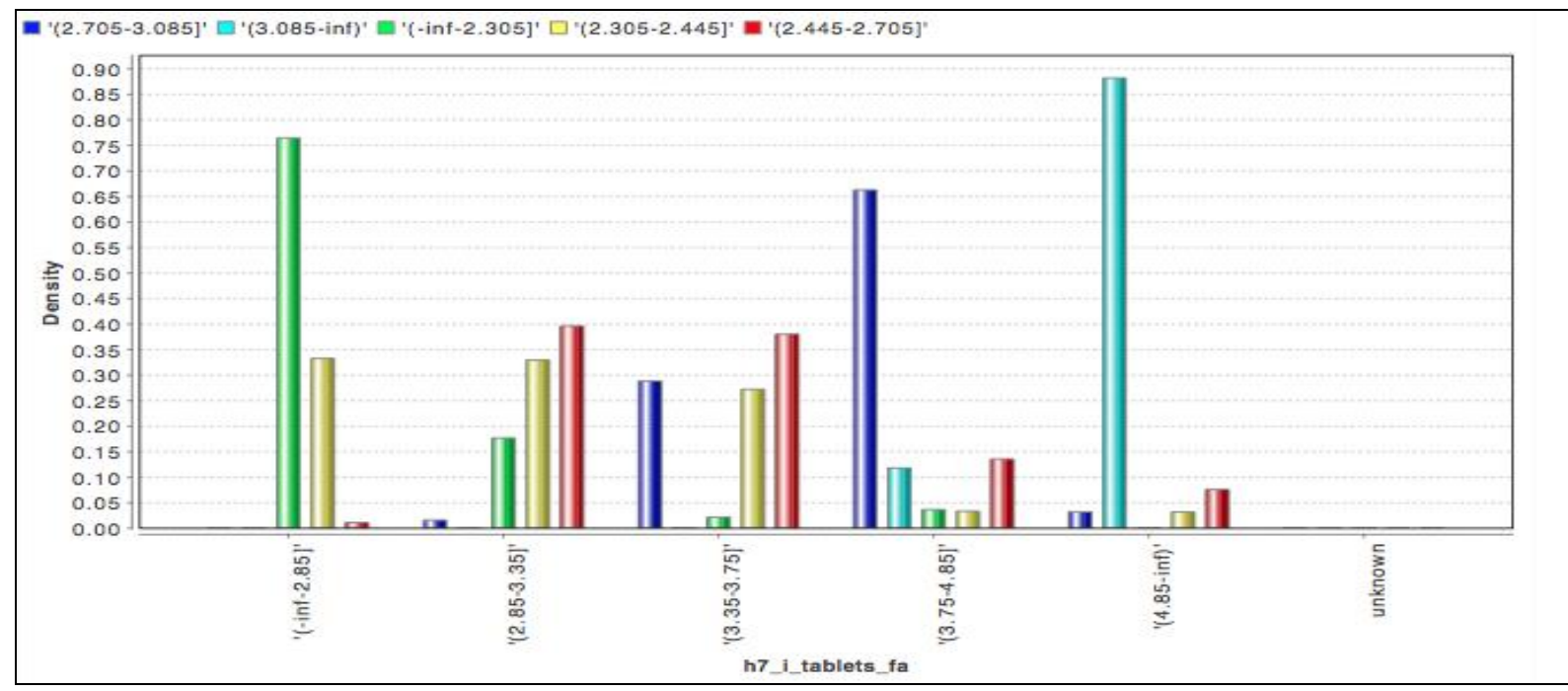

Figura 10. Densidad de probabilidad de intervalos discretos de THDv con relación a la corriente del $7^{\circ}$ armónico.

\section{5 - CONCLUSIÓN}

Sobre la base de los análisis realizados mediante la técnica de prospección de datos (naivebayes) en una base de datos discretos de frecuencia se obtuvieron las siguientes conclusiones: el orden de armónico del $5^{\mathrm{a}}$ al $7^{\mathrm{a}}$ del compresor representó los siguientes porcentajes, $84 \%$ y $88,5 \%$; en las central de aire acondicionado los armónico de orden $7^{\mathrm{a}}$ y $9^{\mathrm{a}}$ representaron los porcentajes de $87,2 \%$ y $83 \%$ respectivamente; en líneas de producción de tablets los armónicos de orden $3^{\mathrm{a}}, 5^{\mathrm{a}}$ 
Waterloo Ferreira da Silva et al. / ITEGAM-JETIA Vol. 01, Nº2, pp.45-54. Junho, 2015.

y $7^{\mathrm{a}}$ tuvieron los menores porcentajes siendo de $82,9 \%, 82,5 \%$ y $88 \%$, en ese orden. Así, los armónicos que más contribuyeron al aumento de la distorsión armónica se encuentran en las líneas de producción de tablets debido a que lo equipamientos de esta línea emplean fuentes de conmutación que se presentan como cargas no lineales generando un desequilibrio entre las fases. En el contexto general el $7^{a}$ armónico tuvo la mayor influencia en el impacto de armónicos, lo que reafirma la preocupación de las recomendaciones de las normas internacionales y la PRODIST [9] en el módulo 8 que hacen referencia a la calidad de energía en los sistemas eléctricos industriales.

\section{6 - Agradecimientos}

A la Universidad Federal de Pará - UFPA, al Instituto de Tecnología Galileo de la Amazonía - ITEGAM, al Instituto de Tecnología José Rocha Sérgio Cardoso - ITJC y Eletrobras Amazonas Energía por el soporte durante la investigación.

\section{7 - Referencias Bibliograficas}

[1] Oliveira, C. G. et al. Estudo de caso de eficiência energética e qualidade de energia elétrica. 6p. Anais do VIII Induscon Conferência Internacional de Aplicações Industriais, Poços de Caldas/MG, 2008.

[2] Nunes, Renato VINHAL. Análise da penetração harmônica em redes de distribuição desequilibradas devido às cargas residenciais e comerciais com a utilização do ATP. Dissertação de Mestrado, PPGEE, UFMG. 2007.

[3] Arrilaga J and N.R. Watson, Power Systems Harmonics, Wiley, 2nd Edition, 2003.

[4] Dugan, R. C.; MCdermott, T.E. Operanting conflits for distributed generation on distribution systems. IEEE. 2001.

[5] Dugan. Roger C. Mark F. MCgranaghan, Surya Santoso, H. Wayne Beaty. Electrical Power Systems Quality. Second Edition. McGraw-Hill. USA. 2004.

[6] IEEE Stardard 519-1992. Recommended Practices and Requirements for Harmonic Control in electrical Power Systems. 1992.

[7] IEC61000-2-2. Electromagnetic compatibility (EMC) - Part 2-2: Environment - Compatibility levels for low-frequency conducted disturbances and signalling in public low-voltage power supply systems.

[8] IEC (IEC 61000-4-7, 61000-4-30) International Electrotechnical Commission.

[9] IEC 61000-4-30. International Electrotechnical Commission. IEC 61000-4-30 Ed. 2.0: Electromagnetic Compatibility (EMC) -
Part 4 - 30: Testing and Measurements Techniques - Power Quality Measurement Methods, 2008.

[10] ANEEL. Módulo 8- Procedimentos de Distribuição de Energia Elétrica no Sistema Elétrico Nacional - PRODIST. 2010.

[11] ANEEL. Nota Técnica, $\mathbf{n}^{\circ} \mathbf{0 0 8 3}$ /2012-SRD/ANEEL, de 12 Junho 2012. Processo: 48500.002798/2012-61.

[12]R. Dugan, M. McGranaghan, H. Wayne, "Electrical Power Systems Quality”, 265 pp., McGraw-Hill, New York, USA. 1999.

[13]IEEE Task Force on Effects of Harmonics on Equipment: "Effects of Harmonics on Equipment", IEEE Transactions on Power Delivery, vol. 8, n. ${ }^{\circ}$ 2, pp. 672-680, April 1993.

[14] Yin, Robert K. Case Study Research: Design and Methods. 4a Edition. Sage Publications of London, Thousand Oaks and New Delhi and Singapure. 2010.

[15] E. Frank, M. Hall, and L. Trigg, "Weka 3-Data Mining with Open Source Machine Learning Software in Java," The University of Waikato, 2000.

[16] H. Bakhtaet all. Naïve possibilistic network classifiers, Fuzzy Sets and Systems. 160 Science Direct (2009) 3224-3238.

[17] Tan, Pang-Ning; Steinbach, Michael; KUMAR, Vipin Introdução ao DATAMINING Mineração de Dados. Rio de Janeiro: Editora Ciência Moderna Ltda., 2009.

[18] P. Langley, W. Iba, K. Thompson, An analysis of Bayesian classifiers, in: Proc. AAAI-92, 1992, pp. 223-228.

[19] P. Langley, S. Sage. Induction of selective Bayesian classifiers, in: Proc. 10th Conf. on Uncertainty in Artificial Intelligence UAI94, 1994, pp.399-406.

[20] D. Grossman, P. Dominigos, Learning Bayesian maximizing conditional likelihood, in: Proc. on Machine Learning, Canada, 2004, pp. 46-57.

[21] R. Duda, P. Hart, Pattern Classification and Scene Analysis, Wiley, New York. 